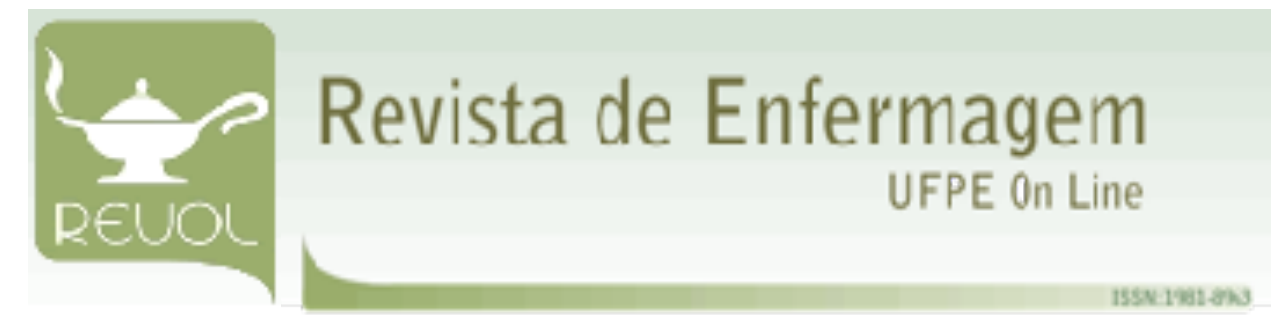

Rev enferm UFPE on line. 2021;15(2):e244597 DOI:

\title{
RISCO DE LESÕES POR PRESSÃO EM IDOSOS NO DOMICÍLIO
}

\author{
RISK OF PRESSURE ULCERS IN THE ELDERLY AT HOME \\ RIESGO DE LESIONES POR PRESIÓN EN ANCIANOS DEL HOGAR
}

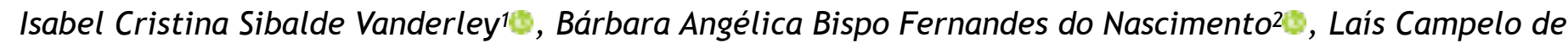
Morais $^{3}$, Camilla Valeriano Cazuza de Souza ${ }^{4}$, Glacineide Cecília dos Santos ${ }^{5}$, Germanna Yamina Ribeiro de Sousa Moraes 6 , Sandra Brotto Furtado Ehrhardt 7

\section{RESUMO}

Objetivo: analisar os fatores associados ao risco de desenvolvimento de Lesões por Pressão nos idosos atendidos pelo Serviço de Atenção Domiciliar. Método: trata-se de um estudo quantitativo, descritivo, do tipo transversal, realizado no Serviço de Atenção Domiciliar com pessoas maiores de 60 anos. Realizou-se a coleta dos dados por meio de questionário sociodemográfico e clínico semiestruturado e Escala de Braden. Analisaram-se os dados a partir do Teste Exato de Fisher e a Razão de Verossimilhança com nível de significância de 5\%. Resultados: observaram-se associações entre o risco de desenvolvimento de Lesão por Pressão e a escolaridade $(p=0,001)$, renda $(p=0,024)$, idosos acamados $(p<0,001)$, portadores de Síndrome de Imobilidade $(p<0,001)$, diagnóstico de úlcera vascular $(p<0,001)$, Acidente Vascular Encefálico $(p=0,009)$ e Demência $(p<0,001)$. Conclusão: evidenciou-se que diferentes fatores interferem no risco de desenvolvimento de Lesão por Pressão nos idosos, sobretudo como resultado de fatores individuais e sociais. Descritores: Lesão por Pressão; Idoso; Saúde do Idoso; Assistência Domiciliar; Serviços de Assistência Domiciliar; Enfermagem Domiciliar.

\section{ABSTRACT}

Objective: to analyze the factors associated with the risk of developing Pressure Ulcers in the elderly assisted by the Home Care Service. Method: This is a quantitative, descriptive, cross-sectional study, carried out at the Home Care Service with people over 60 years old. Data was collected using a semi-structured sociodemographic and clinical questionnaire and the Braden Scale. The data was analyzed using the Fisher's Exact Test and the Likelihood Ratio with a significance level of 5\%. Results: associations were observed between the risk of developing Pressure Ulcers and education $(p=0.001)$, income $(p=0.024)$, bedridden elderly $(p<0.001)$, patients with immobility syndrome 
$(p<0.001)$, diagnosis of vascular ulcer $(p<0.001)$, stroke $(p=0.009)$ and dementia $(p<0.001)$. Conclusion: it was evidenced that different factors interfere in the risk of developing Pressure Ulcers in the elderly, mainly as a result of individual and social factors. Descriptors: Pressure Ulcer; Aged; Health of Elderly; Home Care; Home Care Services; Home Nursing.

\section{RESUMEN}

Objetivo: analizar los factores asociados al riesgo de desarrollar Lesiones por Presión en los ancianos atendidos por el Servicio de Atención Domiciliaria. Método: se trata de un estudio cuantitativo, descriptivo, transversal, realizado en el Servicio de Atención Domiciliaria con personas mayores de 60 años. Los datos se recogieron mediante un cuestionario sociodemográfico y clínico semiestructurado y la Escala de Braden. Los datos se analizaron mediante la prueba exacta de Fisher y la razón de verosimilitud con un nivel de significancia del 5\%. Resultados: hubo asociaciones entre el riesgo de desarrollar Lesión por Presión y educación $(p=0,001)$, ingresos $(p=0,024)$, ancianos postrados en cama ( $p<0,001)$, pacientes con Síndrome de Inmovilidad ( $<<0,001)$, diagnóstico de úlcera vascular $(p<0,001)$, derrame cerebral vascular $(p=0,009)$ y demencia $(p<0,001)$. Conclusión: se evidenció que diferentes factores interfieren en el riesgo de desarrollar Lesión por Presión en el adulto mayor, principalmente como resultado de factores individuales y sociales. Descriptores: Úlcera por Presión; Anciano; Salud del Anciano; Atención Domiciliaria de Salud; Servicios de Atención de Salud a Domicilio; Cuidados de Enfermería em el Hogar.

1Universidade Federal de Pernambuco/UFPE. Recife (PE), Brasil. $1 \oplus$ https://orcid.org/ 0000-0003-0372-9481 2Universidade de Pernambuco/UPE. Recife (PE), Brasil. 2@ https://orcid.org/ 0000-0002-5650-350X 3,4,5,6,7Instituto de Medicina Integral Professor Fernando Figueira. ${ }^{3 \oplus}$ https:// orcid.org/0000-0002-0316-5498 ${ }^{4}$ https://orcid.org/0000-0003-2635-3202 ${ }^{5}$ https://orcid.org/ 0000-0003-1403-7907 6 https://orcid.org/0000-0002-2204-9097 70 https://orcid.org/ $\underline{0000-0003-4285-1009}$

*Artigo extraído de Trabalho de Conclusão de Residência em Saúde do Idoso << Especialização em Saúde do Idoso. Instituto de Medicina Integral Professor Fernando Figueira /IMIP, 2018

\section{Como citar este artigo}

Vanderley ICS, Santana ADS, Nascimento BABF, Morais LC, Souza CVC, Santos GC, et al. Risco de Lesões Por Pressão em idosos no domicílio. Rev enferm UFPE on line. 2021;15(2):e244597 DOI: https://doi.org/10.5205/1981-8963.2021.244597 


\section{INTRODUÇ̃̃O}

Evidencia-se que o envelhecimento populacional é um fato na realidade atual e traz a necessidade de uma reformulação no modelo de atenção à saúde para atender às demandas deste grupo etário. Surge-se, assim, a Atenção Domiciliar (AD) como uma modalidade de assistência substitutiva ou complementar às outras modalidades, que garante a continuidade do cuidado por meio de ações de promoção da saúde, prevenção, tratamento e reabilitação, por meio de visita domiciliar e consulta dos profissionais integrantes da equipe. ${ }^{1}$

Explica-se que os serviços de assistência domiciliar surgiram a partir das dificuldades observadas pelos pacientes no acesso aos demais serviços de saúde. Destacam-se, frente a essas dificuldades, algumas situações clínicas comuns no cotidiano da AD, dentre elas, o risco de desenvolvimento de Lesões por Pressão (LPP). ${ }^{2}$

Identificam-se as LPP na pele e/ou tecido subjacente, que surgem sobre proeminências ósseas e/ ou locais com adiposidade subcutânea diminuída, resultantes da pressão extrínseca. Discute-se que o avanço da idade é um fator que influencia o desenvolvimento dessas lesões, pois são verificadas mudanças tanto na textura quanto nas propriedades da pele com o envelhecimento. Evidencia-se que estas lesões costumam ocorrer em idosos frágeis com mobilidade prejudicada, tornando imprescindível a atuação multiprofissional. ${ }^{3}$ Destaca-se que o aparecimento de LPP impacta negativamente a funcionalidade desses idosos e o seu convívio social. 4

Discute-se que as escalas que avaliam o risco de desenvolvimento das LPP são importantes para a identificação e o direcionamento dos cuidados necessários. Desenvolveu-se a Escala de Braden em 1987 e a mesma foi traduzida e validada para o português em 1999, sendo um instrumento amplamente utilizado no país, avaliando os seguintes aspectos: percepção sensorial; umidade; atividade; mobilidade; nutrição e fricção e cisalhamento. ${ }^{5}$

Notabiliza-se que são escassos os estudos que abordam a temática de LPP em pacientes atendidos em domicílio, 6 denotando a necessidade de expansão nas pesquisas acerca deste assunto, a fim de trazer subsídios para propostas de intervenções eficazes no combate ao desenvolvimento das referidas lesões por meio de sugestão de estratégias de prevenção. Destaca-se que a realização de estudos que abordem o risco de desenvolvimento de LPP em idosos acompanhados no domicílio é importante para o aprimoramento da assistência de Enfermagem e da equipe multiprofissional na AD. 


\section{OBJETIVO}

Analisar os fatores associados ao risco de desenvolvimento de Lesões por Pressão segundo a Escala de Braden nos idosos atendidos pelo Serviço de Atenção Domiciliar (SAD).

\section{MÉTODO}

Trata-se de um estudo quantitativo, descritivo, do tipo transversal, realizado no município de Recife (PE), no SAD do Instituto de Medicina Integral Professor Fernando Figueira (IMIP), no período de abril de 2017 a maio de 2018.

Compõe-se a população do estudo por pessoas maiores de 60 anos acompanhadas pelo SAD do IMIP e com uma amostra por conveniência após o atendimento dos critérios de inclusão e exclusão. Incluíram-se as pessoas com mais de 60 anos atendidas pelo SAD do IMIP. Excluíram-se os idosos com comprometimento cognitivo grave sem a presença de um responsável capaz de fornecer as informações do questionário no momento da entrevista.

Realizou-se a coleta dos dados mediante entrevistas domiciliares, sendo utilizado um questionário semiestruturado, com questões referentes às características sociodemográficas e clínicas dos idosos, além da aplicação da Escala de Braden para avaliar o risco de desenvolvimento de LPP nos idosos da amostra. Aplicou-se o questionário pela pesquisadora e mais cinco enfermeiras do SAD previamente orientadas e treinadas para a aplicação dos questionários.

Validaram-se os dados por dupla digitação em planilhas do Microsoft Excel, exportando-os para o Statistical Package for Social Sciences (SPSS), versão 20.0. Realizou-se a análise a partir da geração de relatórios estatísticos e foram utilizados o Teste Exato de Fisher e a Razão de Verossimilhança com nível de significância de 5\%. Considerou-se o valor de $p-<0,05$.

Ressalta-se que este estudo foi aprovado pelo Comitê de Ética em Pesquisa (CEP) do IMIP sob o número CAAE 71609117.4.0000.5201 em cumprimento à Resolução n ${ }^{\circ} 466 / 2012$ do Conselho Nacional de Saúde (CNS), que trata das diretrizes e normas regulamentadoras de pesquisa envolvendo seres humanos. Destaca-se que todos os envolvidos na pesquisa (idosos e/ou cuidadores) assinaram o Termo de Consentimento Livre e Esclarecido (TCLE) para confirmar a participação no estudo.

\section{RESULTADOS}

Compõe-se a amostra por 97 idosos com idades de 60 a 99 anos, com média de 76,71 de anos. Apresentam-se, na tabela 1 , os resultados relativos às características sociodemográficas dos participantes. 
Tabela 1. Características sociodemográficas da amostra ( $n=97)$. Recife (PE), Brasil. 2018.

\begin{tabular}{|c|c|c|}
\hline Variáveis & $\mathrm{n}$ & $\%$ \\
\hline \multicolumn{3}{|l|}{ Sexo } \\
\hline Masculino & 39 & 40,2 \\
\hline Feminino & 58 & 59,8 \\
\hline \multicolumn{3}{|l|}{ Faixa etária (anos) } \\
\hline 60 a 69 & 27 & 27,8 \\
\hline 70 a 79 & 33 & 34,0 \\
\hline 80 a 89 & 37 & 38,1 \\
\hline \multicolumn{3}{|l|}{ Estado civil } \\
\hline Solteiro(a) & 28 & 28,9 \\
\hline Casado(a) & 31 & 32,0 \\
\hline Divorciado(a) & 04 & 4,0 \\
\hline Viúvo(a) & 34 & 35,1 \\
\hline \multicolumn{3}{|l|}{ Escolaridade } \\
\hline Sem escolaridade & 21 & 21,6 \\
\hline Fundamental incompleto & 55 & 56,7 \\
\hline Fundamental & 08 & 8,2 \\
\hline Médio & 09 & 9,3 \\
\hline Superior & 04 & 4,1 \\
\hline \multicolumn{3}{|l|}{ Ocupação } \\
\hline Aposentado & 51 & 52,6 \\
\hline Benefício de Prestação Continuada & 41 & 42,3 \\
\hline Sem renda & 5 & 5,2 \\
\hline \multicolumn{3}{|l|}{ Renda familiar } \\
\hline Até 1 salário-mínimo & 9 & 9,3 \\
\hline De 2 a 3 salários-mínimos & 86 & 88,6 \\
\hline Mais de 3 salários-mínimos & 2 & 2,1 \\
\hline
\end{tabular}

Evidenciou-se que o diagnóstico clínico mais prevalente foi o Acidente Vascular Encefálico (AVE), com $35,1 \%$, seguido por demência $(30,9 \%)$, úlcera vascular $(23,7 \%)$ e câncer $(14,4 \%)$. Percebeu-se, com relação à classificação de risco da amostra, uma prevalência de risco muito elevado, com $39,2 \%$, elevado $(22,7 \%)$, moderado $(12,4 \%)$, leve $(16,5 \%)$ e sem risco, com $9,3 \%$. Encontrou-se que mais da metade dos idosos estudados estava acamada (54,6\%). Identificaram-se a presença de Sín- 
drome de Imobilidade em $20,6 \%$ e a presença de LPP em $22,7 \%$ da amostra, sendo $18,6 \%$ com lesões em região sacral e 6,2\%, em região trocantérica.

Observa-se, na tabela 2, que o percentual de idosos com risco muito elevado foi maior entre aqueles que tinham o Ensino Fundamental incompleto $(52,7 \%)$. Identifica-se, em relação à renda familiar, que, dos nove idosos que possuíam renda de até um salário-mínimo, quatro foram classificados pela Escala de Braden como risco muito elevado $(44,4 \%)$, três, como risco leve $(33,3 \%)$ e os outros dois, sem risco $(22,2 \%)$, enquanto aqueles dois idosos que referiram renda maior que três salários-mínimos foram ambos classificados como risco leve.

Tabela 2. Avaliação da Escala de Braden segundo as variáveis sociodemográficas, Recife (PE),

Brasil. 2018.

\begin{tabular}{|c|c|c|c|c|c|c|}
\hline Variáveis & $\begin{array}{l}\text { Sem Risco } \\
\mathrm{n}(\%)\end{array}$ & $\begin{array}{l}\text { Leve } \\
\mathrm{n}(\%)\end{array}$ & $\begin{array}{l}\text { Moderado } \\
\mathrm{n}(\%)\end{array}$ & $\begin{array}{l}\text { Elevado } \\
\mathrm{n}(\%)\end{array}$ & $\begin{array}{l}\text { M u i t o } \\
\text { Elevado } \\
\mathrm{n}(\%)\end{array}$ & Valor de $\mathrm{p}$ \\
\hline \multicolumn{7}{|l|}{ Sexo } \\
\hline Masculino & $2(5,3)$ & $5(13,2)$ & $3(7,9)$ & $11(28,9)$ & $17(44,7)$ & $p^{(1)}=0,432$ \\
\hline Feminino & $7(11,9)$ & $11(18,6)$ & $9(15,3)$ & $11(18,6)$ & $21(35,6)$ & \\
\hline \multicolumn{7}{|l|}{ Faixa etária } \\
\hline 60 a 69 & $1(3,7)$ & $2(7,4)$ & $4(14,8)$ & $5(18,5)$ & $15(55,6)$ & \\
\hline 70 a 79 & $3(9,1)$ & $4(12,1)$ & $4(12,1)$ & $9(27,3)$ & $13(39,4)$ & $p^{(1)}=0,315$ \\
\hline 80 a 89 & $5(13,5)$ & $10(27,0)$ & $4(10,8)$ & $8(21,6)$ & $10(27,0)$ & \\
\hline \multicolumn{7}{|l|}{ Estado civil } \\
\hline Solteiro(a) & $2(7,1)$ & $6(21,4)$ & $3(10,7)$ & $4(14,3)$ & $13(46,4)$ & \\
\hline Casado(a) & $3(9,7)$ & $3(9,7)$ & $3(9,7)$ & $9(29,0)$ & $13(41,9)$ & $\mathrm{p}^{(1)}=0,648$ \\
\hline Divorciado(a) & $0(0,0)$ & $2(50,0)$ & $1(25,0)$ & $1(25,0)$ & $0(0,0)$ & \\
\hline Viúvo(a) & $4(11,8)$ & $5(14,7)$ & $5(14,7)$ & $8(23,5)$ & $12(35,3)$ & \\
\hline \multicolumn{7}{|l|}{ Escolaridade } \\
\hline $\begin{array}{l}\text { Sem escolaridade } \\
\text { Fundamental }\end{array}$ & $1(4,8)$ & $2(9,5)$ & $4(19,0)$ & $7(33,3)$ & $7(33,3)$ & \\
\hline incompleto & $3(5,5)$ & $12(21,8)$ & $6(10,9)$ & $5(9,1)$ & $29(52,7)$ & $\mathrm{p}^{(2)}=0,001^{*}$ \\
\hline \multicolumn{7}{|l|}{ Fundamental } \\
\hline \multirow[t]{2}{*}{ Médio } & $2(25,0)$ & $1(12,5)$ & - & $4(50,0)$ & $1(12,5)$ & \\
\hline & $3(33,3)$ & $1(11,1)$ & $2(22,2)$ & $2(22,2)$ & $1(11,1)$ & \\
\hline
\end{tabular}




\section{Ocupação}

Aposentado

$5(9,8) \quad 9(17,6) \quad 6(11,8) \quad 16(31,4) \quad 15(29,4)$

Benefício de

P r e s t a ç ã o 4(9,8) 7(17,1) 6(14,6) $\quad 5(12,2) \quad 19(46,3) \quad p^{(1)=0,336}$

Continuada

Sem renda

$1(20,0) \quad 4(80,0)$

Renda familiar

Até 1 salário-mínimo

$2(22,2) \quad 3(33,3)$

$4(44,4)$

De 2 a 3 salários-

mínimos

$p^{(1)}=0,024^{*}$

Mais de 3 salários-

$7(8,1) \quad 11(12,8)$

$12(14,0)$

$22(25,6)$

$34(39,5)$

mínimos

2(100,0)

Legenda: (1)Teste Exato de Fisher; (2)Razão de Verossimilhança; (*)Associação significativa de 5,0\%.

Avalia-se, na tabela 3, a associação significativa entre a classificação da Escala de Braden e as seguintes variáveis: úlcera vascular; AVE; câncer; demência; idoso acamado; Síndrome de Imobilidade; LPP em região sacral e LPP em região trocantérica. Observa-se que a classificação de risco na Escala de Braden foi estatisticamente significante naqueles idosos acamados $(p<0,001)$, portadores de Síndrome de Imobilidade $(p<0,001)$, com diagnóstico de úlcera vascular $(p<0,001)$, de AVE com $p=0,009$ e de demência $(p<0,001)$.

Tabela 3. Avaliação da Escala de Braden segundo as variáveis clínicas. Recife (PE) Brasil. 2018.

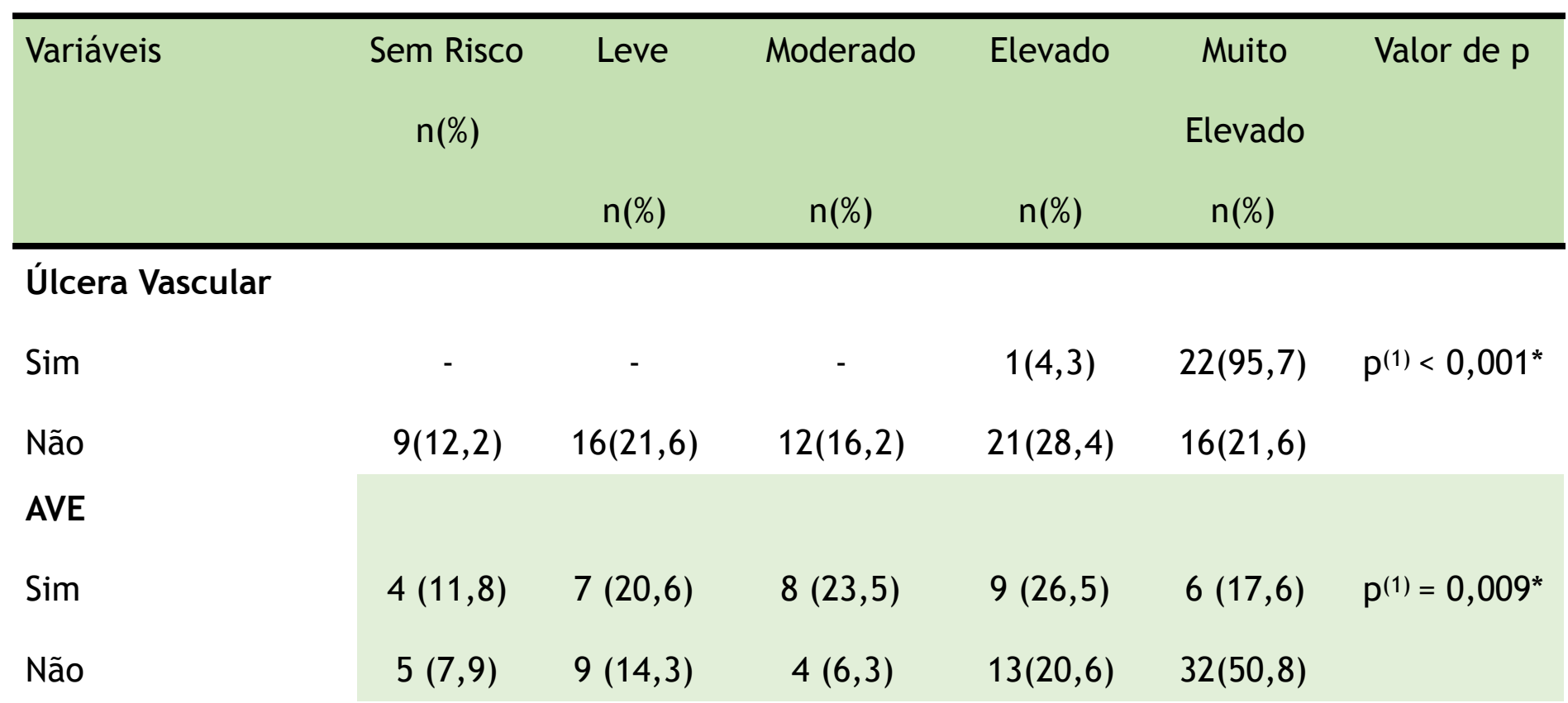




\section{Câncer}

$\operatorname{Sim}$

$2(14,3) \quad 1(7,1)$

$1(7,1)$

$3(21,4)$

$7(50,0)$

$p^{(1)}=0,744$

Não

$7(8,4)$

15(18,1)

$11(13,3)$

19(22,9) $\quad 31(37,3)$

Demência

Sim

$5(16,7) \quad 9(30,0) \quad 5(16,7) \quad 10(33,3) \quad 1(3,3) \quad \mathrm{p}^{(1)<0,001^{*}}$

Não

$4(6,0) \quad 7(10,4) \quad 7(10,4) \quad 12(17,9) \quad 37(55,2)$

Acamado

$\operatorname{Sim}$

$9(17,0) \quad 16(30,2)$

$11(20,8) \quad 14(26,4)$

$3(5,7) \quad \mathrm{p}^{(1)}<0,001^{*}$

Não

$1(2,3) \quad 8(18,2) \quad 35(79,5)$

Síndrome de

Imobilidade

Sim

$6(30,0) \quad 9(45,0) \quad 5(25,0)$

Não

$3(3,9) \quad 7(9,1) \quad 7(9,1) \quad 22(28,6) \quad 38(49,4) \quad p^{(1)}=0,005^{*}$

Lesão por Pressão

Sacral

$\begin{array}{lcccccc}\text { Sim } & \begin{array}{l}3(16,7) \\ \text { Não }\end{array} & 7(38,9) & 1(5,6) & 5(27,8) & 2(11,1) & \\ \text { Lesão por Pressão } & 6(7,6) & 9(11,4) & 11(13,9) & 17(21,5) & 36(45,6) & p^{(1)=0,018^{*}} \\ \text { Trocantérica } & & & & & & \\ \text { Sim } & & & & & & \\ \text { Não } & 2(33,3) & 3(50,0) & - & - & 1(16,7) & p^{(1)}=0,018^{*} \\ & 7(7,7) & 13(14,3) & 12(13,2) & 22(24,1) & 37(40,7) & \end{array}$

Legenda: (1)Teste Exato de Fisher; $\left(^{*}\right)$ Associação significativa de 5,0\%.

\section{DISCUSSÃO}

Evidencia-se a predominância do sexo feminino neste estudo. Verifica-se que este resultado vem sendo encontrado em estudos que avaliaram pacientes domiciliares, 4,6,7-8 o que enfatiza a ocorrência no Brasil, como no mundo, do fenômeno da feminização do envelhecimento, relacionado a uma maior mortalidade masculina, reduzindo a proporção de homens idosos.9-10

Percebe-se que a média de idade dos participantes neste estudo, de 76,7 anos, é menor que em outros estudos em que se observa a predominância de idosos maiores de 80 anos. ${ }^{5,8,11}$ Nota-se, na categorização de faixas etárias, a maior prevalência entre os maiores de 80 anos e $72 \%$ da amostra estava acima de 70 anos. Acredita-se que essa redução na média de idade foi devido à alta prevalência de idosos jovens, entre 60 e 69 anos, que compuseram cerca de um terço da amostra. 
Encontra-se, na variável estado civil, consonância com uma pesquisa desenvolvida em 2016 com idosos na atenção básica, ${ }^{8}$ porém, diferindo dos achados de um estudo realizado em 201412 com idosos hospitalizados, que encontrou uma maior prevalência de casados. Explica-se, possivelmente, esta divergência pela diferença nos desenhos dos estudos e características das amostras. Observouse, nesse estudo, que $32,0 \%$ eram casados e $68,0 \%$ não possuíam cônjuges por serem solteiros, viúvos ou divorciados, alertando a transferência da responsabilidade do cuidado para os filhos, principalmente, que gera repercussões econômicas e sociais por impactar a saída do mercado de trabalho desses jovens cuidadores e no novo papel de cuidador de seus genitores.

Convergem-se os dados em relação à escolaridade com estudos que mostraram maior predominância de idosos com Ensino Fundamental incompleto ou baixa escolaridade.6,8,11-12 Encontrou-se associação significativa entre idosos que possuíam Ensino Fundamental incompleto e risco muito elevado de desenvolvimento de LPP. Elenca-se, além disso, uma prevalência alta de analfabetos (21,6\%), que, se somados estes com aqueles com Ensino Fundamental incompleto $(56,7 \%)$, perfazem $78,3 \%$ da amostra com baixa escolaridade, refletindo como essa geração de idosos brasileiros não teve oportunidade de estudo e, dessa forma, convive, no seu presente, com um envelhecimento sem uma remuneração adequada ao seu sustento.

Divergem-se os dados encontrados quanto à renda familiar em relação a outros estudos que demonstraram uma maior prevalência de renda entre um e dois salários-mínimos.6,8,12 Infere-se que a abordagem do quesito renda tenha sido inadequada, uma vez que, ao abordar a renda familiar, se avalia não só a renda do idoso, mas a do domicílio. Constata-se que $42,0 \%$ da amostra recebe o Benefício de Prestação Continuada (BPC), refletindo a necessidade do auxílio do governo, 52,0\% são aposentados e 4,0\% não possuem renda, o que mostra que a renda do idoso isolada esteja sim em consonância com a verificada em outros estudos.6,11-12 Explica-se, dessa forma, que, ao avaliar a renda por domicílio (familiar) e os custos com o idoso dependente, a faixa entre dois e três salários-mínimos ainda parece insuficiente para prover as necessidades gerais de uma família.

Encontraram-se resultados semelhantes no que se refere aos diagnósticos mais prevalentes quando comparados aos de outros estudos brasileiros realizados em domicílio, os quais evidenciaram, dentre eles, AVE e demências. ${ }^{5,6-8,11}$

Menciona-se que o diagnóstico de AVE foi estatisticamente significante para o risco de LPP $(p=0,009)$ e a associação entre este e a Escala de Braden demonstra percentuais progressivos da ausência de risco $(11,8 \%)$ para riscos leve $(20,6 \%)$, moderado $(23,5 \%)$ e elevado $(26,5 \%)$, o que está de acordo com pesquisas que relacionaram a presença de AVE como predisposição para o surgimento dessas lesões. Observou-se, porém, nesse estudo, uma redução do percentual de risco elevado em comparação com risco muito elevado $(17,6 \%) .5,8,11$ Infere-se que isto ocorra devido às inúmeras sín- 
dromes clínicas e sequelas motoras associadas às não motoras causadas pelo comprometimento vascular em diversas regiões cerebrais e de diferentes amplitudes, causando uma ampla variedade clínica entre os pacientes e diferentes graus de dependência, que são enquadrados no mesmo diagnóstico de AVE, podendo apresentar, então, riscos diversos para o desenvolvimento de LPP.

Verificou-se ainda a associação estatística entre os portadores de diagnóstico de demência e o risco de desenvolvimento de lesões $(p<0,001)$, porém, estes apresentavam, proporcionalmente, $o$ menor percentual de risco muito elevado. Enfatiza-se, então, a importância de destacar que, apesar de não possuir o risco classificado em muito elevado, a maioria dos idosos apresentava riscos de desenvolver a lesão. Sabe-se que a demência é uma doença neurodegenerativa progressiva, que apresenta diferentes fases de evolução e, com isso, afeta, de formas diferentes, a funcionalidade e a mobilidade, gerando riscos menores ou maiores de desenvolvimento de LPP a depender do tempo de diagnóstico da doença.

Identifica-se que os dados referentes ao risco de desenvolver LPP alertam para o impacto das úlceras vasculares na capacidade funcional e na mobilidade do idoso, interferindo sobremaneira no cuidado à saúde. ${ }^{13}$ Associou-se a significativa presença do diagnóstico de úlcera vascular ao risco de desenvolvimento de LPP $(\mathrm{p}<0,001)$, sendo este um fenômeno pouco discutido na literatura e com a necessidade de mais estudos que abordem a temática. Acredita-se que a presença de úlceras vasculares resulte na restrição da mobilidade, proporcionando a permanência do idoso em um estado de repouso mais prolongado, aumentando o risco de desenvolvimento de LPP.

Discute-se que, além da própria imobilidade pela restrição ao leito, os idosos acamados podem evoluir para complicações como tromboses e perda de peso, ${ }^{3}$ aumentando o risco de desenvolvimento de lesões. Aborda-se que uma complicação grave é o surgimento da Síndrome de Imobilidade, que engloba, dentro de seus critérios diagnósticos, o sofrimento cutâneo ou a própria LPP. Sabe-se que a Síndrome de Imobilidade é definida por critérios maiores (déficit cognitivo e múltiplas contraturas) e critérios menores (disfagia, afasia, dupla incontinência e sofrimento cutâneo ou lesão), sendo necessária a presença obrigatória dos dois critérios maiores associados a dois critérios menores, podendo, portanto, o paciente apresentar ou não o diagnóstico de LPP. ${ }^{3}$

Encontraram-se, neste estudo, 54,6\% idosos acamados e que 20,6\% apresentavam Síndrome de Imobilidade e ambos se mostraram associados estatisticamente ao risco de desenvolver LPP $(p<0,001)$. Destaca-se a importância de que as duas condições são conhecidamente fatores de risco para o desenvolvimento destas lesões pela imobilização prolongada.5,8,14 Justificam-se os escores da Escala de Braden para os idosos com Síndrome de Imobilidade por dois motivos: primeiro, os diferentes critérios menores associados ao diagnóstico; segundo, as peculiaridades nos cuidados recebi- 
dos pelos idosos com Síndrome de Imobilidade, que influenciam diretamente o risco de desenvolver LPP.

Elencou-se que, dos 97 idosos avaliados, 22 apresentavam diagnóstico de LPP, correspondendo a uma prevalência de $22,7 \%$. Faz-se necessário ressaltar uma particularidade desta modalidade de serviço de saúde, pois a $A D$ enquadra-se em um serviço de média complexidade, sendo estas lesões, muitas vezes, o motivo de admissão do idoso no programa pela necessidade de diferentes coberturas nos curativos, analgesia e orientações multidisciplinares para o tratamento destas lesões.

Discute-se outro dado encontrado: $18,2 \%$ idosos com LPP não possuíam o risco de desenvolver lesões. Explica-se esse dado por condições extrínsecas como: o desenvolvimento da lesão durante a internação hospitalar (dificuldade de deambulação, colchões recobertos com materiais impermeáveis que aumentam a sudorese e umidade, por exemplo); o desconhecimento da família quanto à mudança de decúbito periódica ou à troca de fraldas frequente ou por características intrínsecas à própria Escala de Braden, que avalia diferentes domínios. ${ }^{6}$

Salienta-se que a localização de maior prevalência das LPP foi as regiões sacral $(81,8 \%)$, trocantérica $(27,3 \%)$ e isquiática $(18,1 \%)$, mencionando que podem vir associadas entre si, corroborando os resultados encontrados em outras pesquisas. ${ }^{6-7}$ Discute-se que uma maior prevalência de LPP na região sacral está relacionada ao prolongado tempo de imobilização no leito, o que sugere um maior risco de desenvolvimento das mesmas devido a uma maior permanência na posição de decúbito dorsal.15-16 Discute-se que, dos 18 idosos com lesões em região sacral, três não apresentavam riscos de desenvolvimento de outras lesões, o que denota a importância de se avaliar o risco, por meio da aplicação de uma escala, mesmo naqueles idosos que já possuam uma lesão. Observou-se que a presença de LPP demonstrou associação significativa para o risco de desenvolvimento de outras lesões $(p<0,001)$ e, quando categorizadas por região, tanto a sacral quanto a trocantérica mantiveram a associação com $\mathrm{p}=0,005$ e $\mathrm{p}=0,018$, respectivamente.

Menciona-se que as limitações do estudo se associam ao desenho metodológico do estudo, pois não permite a avaliação dos idosos do SAD com um recorte temporal maior, além do fato do mesmo ter sido realizado em apenas um serviço de assistência domiciliar, não representando toda a magnitude do problema estudado. Faz-se necessário que outras pesquisas, com outros desenhos metodológicos, sejam desenvolvidas para que se haja um maior aprofundamento nas questões relacionadas ao risco de desenvolvimento de LPP em idosos acompanhados no domicílio.

\section{CONCLUSÃO}

Evidenciou-se a associação de características sociodemográficas e clínicas de idosos atendidos em um SAD em Pernambuco com o risco de desenvolvimento de LPP, avaliando-os pela Escala de 
Braden. Discorre-se que a baixa escolaridade, a presença de úlcera vascular, o idoso acamado, portador de Síndrome da Imobilidade, de AVE e de demência apresentaram associação significativa com o risco de desenvolvimento de LPP.

Ressalta-se que estudos que avaliam o risco de desenvolvimento de LPP na população idosa domiciliada são de grande contribuição e relevância, visto que esta população está em acelerado crescimento e a assistência domiciliar configura-se como um essencial componente substitutivo e/ ou complementar ao modelo hospitalocêntrico.

\section{CONTRIBUIÇÕES}

Informa-se que todos os autores contribuíram igualmente na concepção do projeto de pesquisa, coleta, análise e discussão dos dados, bem como na redação e revisão crítica do conteúdo com contribuição intelectual e na aprovação da versão final do estudo.

\section{CONFLITO DE INTERESSES}

Nada a declarar.

\section{REFERÊNCIAS}

1. Ministério da Saúde (BR). Conselho Nacional de Saúde. Portaria n 825, de 25 de abril de 2016. Redefine a Atenção Domiciliar no âmbito do Sistema Único de Saúde (SUS) [Internet]. Ministério da Saúde; 2016 [cited 2019 Feb 10]; Available from: http://bvsms.saude.gov.br/bvs/saudelegis/gm/ 2016/prt0825_25_04_2016.html

2. Ministério da Saúde (BR). Secretaria de Atenção à Saúde. Departamento de Atenção Básica. Caderno de atenção domiciliar [Internet]. 2013 [cited 2019 Feb 10];2(1):1-205. Available from: http://189.28.128.100/dab/docs/publicacoes/geral/cad_vol1.pdf

3. Freitas EV, Py L. Tratado de geriatria e gerontologia. 4. ed. Rio de Janeiro: Guanabara Koogan, 2016 [cited 2019 Feb 12].

4. Vieira VAS, Santos MDC, Almeida AN, Souza CC, Bernardes MFVG, Mata LRF. Risco de Lesão Por Pressão em idosos com comprometimento na realização de atividades diárias. Rev de Enfermagem do Centro-Oeste Mineiro 2018 [cited 2019 Feb 14]; 8:e2599. DOI: 10.19175/recom.v7i0.2599

5. Santos RR, Zagonel IPS, Sanches LC, Ribeiro ER, Garbeline MCL. Educação em saúde: conhecimento dos enfermeiros para prevenção da lesão por pressão no domicílio. Rev Espaço para a Saúde. 2018 [cited 2019 Mar 10]; 19(2):54-63. DOI: 10.22421/15177130-2018v19n2p54 
6. Moro JV, Caliri MHL. Pressure ulcer after hospital discharge and home care. Escola Anna Nery [Internet]. 2016 [cited 2019 Feb 11]; 20(3):e20160058. DOI: 10.5935/1414-8145.20160058

7. Vieira CPB, Araújo TME. Prevalence and factors associated with chronic wounds in older adults in primary care. Rev Esc Enferm USP. 2018 [cited 2019 Mar 12]; 52:e03415. DOI: 10.1590/ S1980-220X2017051303415

8. Vieira CPB, Oliveira EWF, Ribeiro MGC, Luz MHBA, Araújo OD, Pereira AFM. Preventive actions in pressure ulcers carried out by nurses in primary care. J Res Fundam Care Online [Internet]. 2016 [cited 2019 Feb 11]; 8(2):4447-59. DOI: 10.9789/2175-5361.2016.v8i2.4447-4459

9. Ministério do Planejamento, Orçamento e Gestão (BR), Instituto Brasileiro de Geografia e Estatística. Síntese de indicadores sociais: uma análise das condições de vida da população brasileira: 2018 [Internet]. Rio de Janeiro: IBGE; 2018 [cited 2019 Jan 10]; Available from: https:// biblioteca.ibge.gov.br/visualizacao/livros/liv101629.pdf

10. Rajasi RS, Mathew T, Nujum TZ, Anish TS, Ramachandran R, Lawrence T. Quality of Life and Sociodemographic Factors Associated with Poor Quality of Life in Elderly Women in Thiruvananthapuram, Kerala. Indian Journal of Public Health [Internet]. 2016 [cited 2019 Jan 19]; 60(3). DOI: 10.4103/0019-557X.189016.

11. Ayala ALM, Galende ACBPS, Stoeberl FR. Cuidados de enfermagem na prevenção de úlcera por pressão em pacientes acamados no domicílio. Semina Cienc Biol Saúde [Internet]. 2016 [cited 2019 Apr 14]; 37(2):25-38. DOI: 10.5433/1679-0367.2016v37n2p25

12. Vieira VAS, Santos MDC, Almeida AN, Souza CC, Bernardes MFVGB, Mata LRF. Risco de lesão por pressão em idosos com comprometimento na realização de atividades diárias. Revista de Enfermagem do Centro-Oeste Mineiro. 2018 [cited 2020 jun 28]; 8:e2599. DOI: 10.19175/ recom.v7i0.2599

13. Joaquim FL, Camacho ACLF, Sabóia VM, Santos RC, Santos LSF, Nogueira GA. Impact of home visits on the functional capacity of patients with venous ulcers. Rev Bras Enferm [Internet]. 2016 [cited 2019 Feb 16]; 69(3):468-77. DOI: 10.1590/0034-7167.2016690308i

14. Debon R, Fortes VLF, Rós ACR, Scaratti M. The Nurses' Viewpoint Regarding the Use of the braden Scale With the Elderly Patient. J Res Fundam Care Online [Internet]. 2018 [cited 2019 Jan 11]; 10(3): 817-823. DOI: 10.9789/2175-5361.2018.v10i3.817-823

15. Pedrosa IL, Silva MSML, Araújo AA, Schwanke CHA, Carli GA, Gomes I. Pressure ulcers in elders and in non-elders: a historical cohort study. Online Braz J Nurs [Internet]. 2014 [cited 2019 Feb 11]; 13(1):82-91. DOI: 10.5935/1676-4285.20144111 
16. Oomens CWJ, Broek M, Hemmes B, Bader DL. How does lateral tilting affect the internal strains in the sacral region of bed ridden patients? A contribution to pressure ulcer prevention. Clin Biomech [Internet]. 2016 [cited 2019 Jan 10]; 35:7-13. DOI: 10.1016/j.clinbiomech.2016.03.009

\section{Correspondência}

Isabel Cristina Sibalde Vanderley

E-mail: belvanderley@hotmail.com

Submissão: 21/03/2020

Aceito: $19 / 06 / 2021$

Copyright $\odot 2021$ Revista de Enfermagem UFPE on line.

Este é um artigo de acesso aberto distribuído sob a Atribuição CC BY 4.0 Creative Commons AttributionShareAlike 4.0 International License, a qual permite que outros distribuam, remixem, adaptem e criem a partir do seu trabalho, mesmo para fins comerciais, desde que lhe atribuam o devido crédito pela criação original. É recomendada para maximizar a disseminação e uso dos materiais licenciados. 